\title{
INSTRUMENTOSPSICOLÓGICOSMAISCONHECIDOSEUTILIZADOSPORESTUDANTESE PROFISSIONAISDEPSICOLOGIA
}

\author{
Ana Paula Porto Noronha \\ Flávia Nunes de Moraes Beraldo ${ }^{2}$ \\ Katya Luciane de Oliveira ${ }^{3}$
}

\section{Resumo}

O presente estudo objetivou identificar os instrumentos psicológicos mais conhecidos por estudantes e profissionais de psicologia e levantar os instrumentos mais utilizados pela amostra. Participaram 82 alunos último-anistas, de uma universidade particular do interior paulista e 52 profissionais. O material utilizado constituiu-se de uma relação de instrumentos de avaliação psicológica e a tarefa dos sujeitos era assinalar os instrumentos conhecidos, os utilizados, e os desconhecidos. A aplicação se deu no próprio período de aula, com a respectiva autorização da coordenação e dos professores. Para a coleta dos dados dos profissionais formados, os questionários foram enviados pelo correio, pois o instrumento é auto-aplicável. Os resultados indicaram uma diferença entre os grupos no que diz respeito aos instrumentos mais conhecidos e utilizados por estudantes e profissionais. Estudos comprovam que alguns dos instrumentos mais conhecidos são também os mais freqüientes nos cursos de formação de psicologia. Sugere-se que novas pesquisas desta natureza sejam realizadas.

Palavras Chave: Avaliação psicológica; Instrumentos psicológicos; Testes psicológicos.

\section{Well-know Psychological Instruments used by Psychology Students and Professional}

\begin{abstract}
This study aimed to identify well-know psychology instruments used by psychology students and professionals and list used instruments in the sample. 82 senior students in a private university of São Paulo countryside participated in the study along with 52 professionals. The material used in this study comprised a setoff psychological assessment instruments and the task assigned to subjects was to check know, used and unknown tools. The task was performed during class hours, authorized by teachers and coordination. To collect data from majored professional, questionnaires were sent by mail because it is a self-applied questionnaire. Results indicated a difference between the 2 groups concerning well-know and used instruments by students and professionals. Studies show that some well-known instruments are also the most frequent in psychology courses. New researches on this topic should be done to gather further data.
\end{abstract}

Key words: Psychological assessment; Psychological instruments; Psychological tests.

\section{INTRODUÇÃo}

A valiação psicológica é um processo de coleta de dados, cuja realização inclui métodos e técnicas de investigação. Os testes psicológicos, por sua vez, são instrumentos exclusivos do psicólogo e são úteis à medida que, quando utilizados adequadamente, podem oferecer informações importantes sobre os testandos.

Embora na literatura haja registros de que os primeiros testes simples, com estruturas frágeis, tenham sido criados no final do século XIX e apesar de haver mais de um século de história na área, os instrumentos atuais ainda apresentam falhas e sofrem críticas. Para Almeida (1999) os instrumentos psicológicos não acompanharam o desenvolvimento das demais áreas de conhecimento, como a informática ou a tecnologia, tendo em vista que os instrumentos atuais muito se aproximam dos iniciais.

Em contrapartida, há perspectivas para a superação das dificuldades apresentadas, pois segundo Sisto, Sbardelini e Primi (2001) tal quadro parece estar sendo revertido, considerando que o Conselho Assessor de Psicologia no CNPq definiu a subárea de "Fundamen-

\footnotetext{
${ }^{1}$ Doutora em Psicologia: ciência e profissão pela PUC-Campinas; Docente do Curso de Psicologia e do Programa de Estudos Pós-graduados em Psicologia, da Universidade São Francisco, campus Itatiba-SP.

${ }^{2}$ Psicóloga, mestranda do Programa de Estudos Pós-Graduados em Psicologia da Universidade São Francisco e docente da UNIFENAS.

${ }^{3}$ Psicóloga e mestranda bosista Capes do Programa de Estudos Pós-Graduados em Psicologia da Universidade São Francisco.
} 
tos e Medidas em Psicologia" como uma das cinco, dentre dez existentes, que mereceriam atenção e investimentos, o que pode gerar um avanço na área.

\section{A Formação Profissional em Avaliação Psicológica}

Formar profissionais competentes não é tarefa fácil. A cada ano muitos psicólogos se formam e devem desenvolver atividades pertinentes à sua atuação profissional, o que inclui a realização da avaliação psicológica; tal atividade representa a psicologia e a difunde na sociedade. Portanto é importante que haja esmero neste trabalho e em todos os outros, a fim de que a ciência psicológica seja mais divulgada reconhecida.

No Brasil, os psicólogos se formam nos cursos promovidos por instituições de ensino superior, com duração de dez/doze semestres, para cursos diurnos/noturnos e, segundo Pfromm Netto (1991), a preparação possui os seguintes objetivos: atender às necessidades do profissional para a atuação; proporcionar ao aluno um conjunto amplo e diversificado de conhecimento, habilidades, atitudes e procedimentos; contribuir para com o processo científico e estimular a produção brasileira de conhecimento.

Embora tais objetivos tenham sido traçados para a formação geral em psicologia, também são pertinentes para a formação específica em avaliação ou em qualquer outra área de conhecimento, considerando que o psicólogo avaliador deve estar preparado para as demandas do mercado profissional, deve conhecer profundamente conceitos teóricos e metodológicos fundamentais e deve contribuir para o progresso da avaliação psicológica.

No entanto, na prática, nem sempre é isto que se encontra. Segundo Buettner (1997) "o que observamos é que o aluno sai da universidade sem a competência necessária para o exercício profissional. Os cursos de graduação, mesmo quando propiciam uma boa formação, o que não ocorre com a grande maioria, enfocam uma formação básica e genérica" (p. 16). Witter, Witter, Yukmitsu e Gonçalves (1992) enfocam que a formação universitária do psicólogo muitas vezes não é ideal, evidenciando a necessidade da busca de um curso de pósgraduação que em parte seria remediativo, porém poucos são os profissionais que buscam uma especialização.

Corroborando a afirmação, Cardoso (1994) aponta que a educação superior não deve ser entendida como oportunidade de emprego, tendo em vista a qualificação recebida pelo o aluno durante o curso superior, pois de uma forma geral, os alunos chegam ao final do curso com sérias deficiências nas questões teóricas e metodológicas o que resulta em uma baixa qualificação no mercado profissional.

Atualmente a profissão sofre com a constante concorrência de outros profissionais que atuam nos mais diversificados setores como escolas, empresas entre outros. Em parte o profissional tem uma grande contribuição quanto a esta situação, pois quando do término da graduação, os profissionais não dão continuidade à formação, a fim de melhor qualificação profissional. Um profissional desqualificado contribui para uma imagem negativa da profissão. Dentro desta perspectiva, Witter e cols. (1992) destacam que toda profissão tem uma imagem social e com o psicólogo não poderia ser diferente, sendo que tal imagem é construída pelo profissional e está diretamente relacionada com o respeito, autoridade, confiança e espaço de atuação.

Ainda em relação à formação do psicólogo, no trabalho desenvolvido por Rocha Jr. e Sá (1997) pertinente à análise dos currículos de psicologia, de nove universidades brasileiras, verificou-se que a pesquisa e a extensão deveriam estar mais presentes nos cursos, que a formação se volta mais para ações curativas, que preventivas, que a formação é generalista e que, segundo os alunos entrevistados, o currículo não é integrado.

Discussões sobre a questão da formação do psicólogo na psicologia ou especificamente na área de avaliação psicológica não têm faltado. E, segundo Witter (1996) têm interessado aos pesquisadores nacionais e internacionais, uma vez que vêm consolidar as questões relativas à formação e atuação profissional, para que as práticas profissionais se mantenham críticas, atualizadas e atentas às necessidades sociais. Alguns estudos versam sobre a crença de que os testes deveriam ser ensinados de forma integrada com os outros conceitos psicológicos (Kroeff, 1998). Jacquemin (1995) defende que deve existir uma programação mínima básica para a formação e que seja priorizada a qualidade do ensino, e não a quantidade de testes ensinados; e Hays e Wellard (1998) acreditam que é evidente a necessidade de continuidade dos estudos após a graduação, em relação à área.

Portanto, os estudos revelam a necessária modificação em relação aos currículos existentes nas instituições brasileiras de ensino superior, em relação à metodologia de ensino utilizada (quantidade ou quali- 
dade) e à criação de cursos de pós-graduação na área. Já em relação aos alunos parece também haver necessidade de maior compromisso com a sua preparação profissional, uma vez que estudos revelam que a proficiência em disciplinas de T.E.P. depende de fatores relacionados aos alunos como motivação, freqüência às aulas, participação, capacidade de raciocínio e integração ao ensino superior, dentre outros; e de fatores relacionados à complexidade do conteúdo ensinado (Primi \& Munhóz, 1998).

\section{Testes Psicológicos}

Os testes psicológicos, apesar de se constituírem em instrumentos úteis ao psicólogo, recebem muitas críticas e vêm sendo questionados. Dentre os estudos recentes que se destinaram a estudar o status do instrumento psicológico, destaca-se o de Noronha (1999) que identificou que grande parte da amostra de psicólogos estudada não utiliza testes psicológicos e que dentre os problemas listados, encontra-se a própria fragilidade do material, o uso inadequado dele e a formação profissional insatisfatória em relação à área. Em outro estudo desenvolvido por Azevedo, Almeida, Pasquali e Veiga (1996), o baixo teor científico dos testes foi denunciado, além da urgente necessidade de melhoria. Almeida, Prieto, Muñiz e Bartram (1998) revelaram que usar materiais inadequados para os objetivos da avaliação, xerocar folhas de resposta, realizar avaliações incorretas, não ter clareza das limitações dos instrumentos, usar testes não adaptados para as diferentes realidades, dentre outros, são os problemas mais delatados na prática dos testes.

$\mathrm{Na}$ literatura internacional são frequientes os estudos sobre o tema. Numa consulta ao PsycINFO (19992000) é possível encontrar 27554 artigos na área de psicologia, sendo que 11275 são relativos à testes. Já no trabalho desenvolvido por Alchieri e Scheffel (2000) com o objetivo de documentar e resgatar a produção científica brasileira em periódicos nacionais na área de psicologia, foram encontrados 1090 artigos sobre avaliação psicológica num período de seis décadas (1930-1999).

Como se vê é urgente a necessidade de estudos científicos na área, portanto, tendo em vista as questões destacadas o presente trabalho teve como objetivo avaliar o conhecimento que psicólogos e formandos em psicologia têm a respeito dos instrumentos psicológicos. Além disto, o estudo pretende listar os instrumentos mais utilizados pelos sujeitos na sua prática profissional.

\section{MÉTODO}

\section{Participantes}

Participaram como sujeitos deste estudo 134 indivíduos entre estudantes de psicologia e psicólogos, que foram divididos em dois grupos, a saber:

Grupo I: alunos último anistas do curso de psicologia, de uma instituição de ensino superior particular do interior paulista, representando $61,2 \%(N=82)$ da amostra. A idade variou de 21 a 49 anos, com média 26,41 $(D P=5,31)$. Em relação aos gêneros, $7,32 \%(N=6)$ eram do sexo masculino e $92,68 \%(N=76)$ do sexo feminino;

Grupo II: psicólogos, representando 38,8\% $(N=52)$ da amostra, com idade variando de 23 a 58 anos e média 34,1 $(D P=8,2)$. Os sujeitos do sexo feminino representaram $84,6 \%(N=44)$ da amostra e do sexo masculino, $15,4 \%(N=8)$. Os sujeitos são formados em média há 7,6 anos $(D P=8,0)$.

Vale ressaltar que três sujeitos do grupo I não preencheram os dados de identificação e, portanto, não foram computados nestes aspectos.

\section{Instrumentos}

Para a viabilização da pesquisa foi utilizada uma relação elaborada pelas autoras, contendo instrumentos de avaliação psicológica. Na relação estavam presentes 169 instrumentos das seguintes editoras: 49 do CEPA, 50 da VETOR, 16 da CASA DO PSICÓLOGO, 12 da EDITES, 8 da CETEPP, 1 da ARTES MÉDICAS, 14 testes estrangeiros, 5 da EDITORIAL PSY, 4 da MESTRE JOU, 1 da MELHORAMENTOS, 1 da ENTRELETRAS e 8 testes de editoras não localizadas.

Os sujeitos tinham quatro possibilidades de resposta para cada instrumento listado, a saber: (a) conheci na graduação / pós-graduação; (b) já utilizei; (c) conheci por procura espontânea; (d) desconheço. Não havia na instrução a obrigatoriedade de que o sujeito assinalasse uma única alternativa por instrumento, portanto as respostas que possuíam mais de uma alternativa foram consideradas, desde que não houvesse incoerências, como o assinalamento de " $(a) e(d)$ ".

Além da relação de instrumentos, o material possuía um quadro para os dados de identificação no que se refere aos seguintes aspectos: idade, sexo, área de atuação e tempo de profissão (no caso de profissionais) e, no caso de alunos, idade e sexo. 


\section{Procedimento}

Os instrumentos foram aplicados com a devida autorização dos sujeitos. Houve diferentes tipos de procedimento de acordo com os grupos estudados. Para os alunos, os questionários foram aplicados coletivamente, em horário de aula previamente cedida pelo professor, e com a autorização da coordenadora de curso, sendo que a participação era voluntária. As aplicações aconteceram em três salas distintas, de último ano de curso (turnos matutino e noturno).

Para a coleta dos dados dos profissionais formados, os questionários foram enviados pelo correio. Foram encaminhados para 87 psicólogos e foram devolvidos, 48, o que representou $55,2 \%$ do total. Juntamente com a relação de instrumentos e a carta de apresentação, foi enviado um envelope selado e etiquetado para facilitar a devolução. Vale ressaltar que fizeram parte do grupo de psicólogos formados 14 alunos de pós-graduação da mesma instituição do grupo de estudantes; para os sujeitos do grupo II, a aplicação foi coletiva, nas mesmas condições do grupo I.

\section{Resultados}

Após a coleta de dados, os resultados foram organizados em tabelas, de acordo com os objetivos do estudo. A análise foi realizada a partir da freqüência das respostas e das respectivas porcentagens.

Em relação ao primeiro objetivo (identificar os instrumentos psicológicos mais utilizados pelos estudantes e profissionais de psicologia), os resultados revelaram que, para o grupo I, a média de testes conhecidos foi $37,82(D P=36,87)$ o que indica um conhecimento de $21,82 \%$ da relação apresentada. Os 15 instrumentos com maior frequiência de resposta foram (Tabela 1): Teste de Apercepção Temática - T.A.T. $(97,56 \% ; N=80)$; Raven - Matrizes Progressivas - escala avançada $(95,12 \% ; N=78)$; O Desenho da Figura Humana $(93,9 \%$; $N=77)$; Bender - Teste Gestáltico Viso-Motor $(91,46 \%$; $N=75$ ); Wartegg, Teste de Apercepção Temática para Crianças - C.A.T. (animais) e Raven - Martrizes Progressivas - escala geral $(90,24 \% ; N=74)$; Teste de Apercepção Temática para Crianças - C.A.T. / humanas $(89,02 \% ; N=73)$; Teste de Zulliger $(86,59 \% ; N=71)$; Escala de Maturidade Mental Columbia - CEPA $(81,71 \% ; N=67)$; Teste da Árvore $(80,49 \% ; N=66)$; Teste de Apercepção Temática para Crianças - suplemento animais, Matrizes Progressivas Coloridas e M.M.P.I. $(78,05 \% ; N=64)$ e WISC $(59 \% ; N=71,95)$.
Tabela 1: Freqüência e respectiva porcentagem dos instrumentos mais conhecidos por estudantes de Psicologia

\begin{tabular}{|c|c|c|}
\hline Nome do Instrumento & $\mathbf{F}$ & $\%$ \\
\hline Teste de Apercepção Temática - T.A.T & 80 & 97,56 \\
\hline Raven - escala avançada & 78 & 95,12 \\
\hline O Desenho da Figura Humana & 77 & 93,9 \\
\hline Bender - Teste Gestaltico Viso-Motor & 75 & 91,46 \\
\hline Wartegg & 74 & 90,24 \\
\hline Teste de Apercepção Temática C.A.T- A & 74 & 90,24 \\
\hline Raven - escala geral & 74 & 90,24 \\
\hline Teste de Apercepção Temática C.A.T. - H & 73 & 89,02 \\
\hline Teste Zulliger & 71 & 86,59 \\
\hline Escala de Maturidade Mental Columbia - CEPA & 67 & 81,71 \\
\hline Teste da Árvore & 66 & 80,49 \\
\hline Teste de Apercepção Temática - C.A.T.-A sup. & 64 & 78,05 \\
\hline Matrizes Progressivas Coloridas & 64 & 78,05 \\
\hline MMPI & 64 & 78,05 \\
\hline WISC & 59 & 71,95 \\
\hline Escala de Mat. Mental Columbia - Casa Psicól. & 58 & 70,73 \\
\hline Teste das Fábulas & 58 & 70,73 \\
\hline Rorschach & 58 & 70,73 \\
\hline Teste de Aptidão Mecânica & 53 & 64,63 \\
\hline Fábulas de Düss & 49 & 59,76 \\
\hline $16 \mathrm{PF}-5^{\mathrm{a}}$ edição & 45 & 54,88 \\
\hline WISC III & 44 & 53,66 \\
\hline Bateria Fatorial CEPA & 44 & 53,66 \\
\hline Teste Palográfico & 44 & 53,66 \\
\hline Pré-Bender & 40 & 48,78 \\
\hline PMK & 32 & 39,02 \\
\hline IAR & 32 & 39,02 \\
\hline Teste das Pirâmides das Cores - 14M - Pfister & 30 & 36,59 \\
\hline Inventário de Interesses Angelini e Thurstone & 30 & 36,59 \\
\hline DAT - Teste de Aptidões Específicas & 28 & 34,15 \\
\hline Teste das Pirâmides das Cores - 24M - Pfister & 27 & 32,93 \\
\hline Escala de Beck & 25 & 30,49 \\
\hline G36 - Teste não Verbal de Inteligência & 25 & 30,49 \\
\hline Bender Hutt & 21 & 25,61 \\
\hline G38 - Teste não Verbal de Inteligência & 21 & 25,61 \\
\hline Os contos de fada e a psicopedagogia & 21 & 25,61 \\
\hline Escala Gesell & 20 & 24,39 \\
\hline WAIS & 20 & 24,39 \\
\hline LIP - Levantamento de Interesses Profissionais & 20 & 24,39 \\
\hline Teste das Cores & 19 & 23,17 \\
\hline Escala Intelig. Stanford-Binet (Terman-Merrill) & 19 & 23,17 \\
\hline IFP - Inventário Fatorial de Personalidade & 18 & 21,95 \\
\hline AC - Teste de Atenção Concentrada & 18 & 21,95 \\
\hline RF - Reprodução de Figuras & 17 & 20,73 \\
\hline INV & 16 & 19,51 \\
\hline Teste de Aptidão Mecânica & 16 & 19,51 \\
\hline Teste de Goodenough & 16 & 19,51 \\
\hline Teste Piaget-Head & 16 & 19,51 \\
\hline GEIST - Inventário llustrado de Interesses & 15 & 18,29 \\
\hline IDATE & 15 & 18,29 \\
\hline Inventário Profissional de Interesses & 15 & 18,29 \\
\hline WPPSI & 15 & 18,29 \\
\hline BTAG - Bateria de Testes de Aptidão Geral & 14 & 17,07 \\
\hline Kuder - Inventário de Interesses & 14 & 17,07 \\
\hline Questionário de Orientação Individual & 14 & 17,07 \\
\hline
\end{tabular}




\begin{tabular}{lll} 
Teste de Organização Percepto-Motora & 14 & 17,07 \\
AC15 - Teste de Atenção Concentrada & 14 & 17,07 \\
BPR-5 & 13 & 15,85 \\
CIA & 13 & 15,85 \\
DHP - Teste Diagnóstico Habilidade Pré-escolar & 13 & 15,85 \\
Teste Prontidão Leitura & 13 & 15,85 \\
Test Guest Visomotor (Lauretta Bender) & 12 & 14,63 \\
IAT - Inventário de Atitudes do Trabalho & 12 & 14,63 \\
D48 & 12 & 14,63 \\
IDATE-C & 12 & 14,63 \\
Teste Projetivo Omega & 12 & 14,63 \\
SAT - Teste de Apercepção para Idosos & 11 & 13,41 \\
Teste de Prontidão para Alfabetização & 10 & 12,2 \\
ADT - Inventário de Administração de Tempo & 10 & 12,2 \\
ESI - Escala de Stress Infantil & 10 & 12,2 \\
D2 - Teste de Atenção Concentrada & 10 & 12,2 \\
HTM - Teste Habilidade para Trabalho Mental & 10 & 12,2 \\
QVI - Questionário Vocacional de Interesses & 10 & 12,2 \\
R1 - Teste não Verbal de Inteligência & 10 & 12,2 \\
\hline
\end{tabular}

A média de testes conhecidos do grupo II foi 48,79 ( $D P=18,2$ ), o que indica um conhecimento de $28,87 \%$ da lista de instrumentos apresentada. A Tabela 2 apresenta os instrumentos mais conhecidos pelos sujeitos deste grupo, sendo que os 15 instrumentos mais freqüentemente identificados pelos sujeitos foram: WISC (98,1\%; $N=51)$; Rorschach, Teste de Zulliger e Desenho da Figura Humana (98,1\%; $N=48)$; Raven - escala geral e escala avançada, Bender e Teste de Apercepção Temática $(88,5 \% ; N=46)$; Teste de Wartegg, C.AT. animal e humano e Teste da Árvore (86,5\%; $N=45$ ); WISC III, M.M.P.I. e Escala de Maturidade Mental Columbia $(82,7 \% ; N=43)$.

Tabela 2: Freqüência e respectiva porcentagem dos instrumentos mais conhecidos por psicólogos.

\begin{tabular}{lcc}
\hline Nome do Instrumento & F & $\%$ \\
\hline WISC & 51 & 98,1 \\
Rorschach & 48 & 92,3 \\
Zulliger & 48 & 92,3 \\
O Desenho da Figura Humana & 48 & 92,3 \\
Raven Matrizes Progressivas-escala geral & 47 & 90,4 \\
Raven Matrizes Progressivas-escala avançada & 46 & 88,5 \\
Bender-Teste Gestaltico Visomotor & 46 & 88,5 \\
Teste de Apercepção Temática - T.A.T. & 46 & 88,5 \\
Teste Wartegg & 45 & 86,5 \\
CAT - Animais & 45 & 86,5 \\
CAT - Humanas & 45 & 86,5 \\
Teste da Árvore & 45 & 86,5 \\
WISC III & 43 & 82,7 \\
MMPI & 43 & 82,7 \\
Escala de Mat. Mental Columbia - CEPA & 43 & 82,7 \\
Escala de Mat. Mental Columbia - Casa Psicól. & 42 & 80,8 \\
G-36- Teste não Verbal de Inteligência & 41 & 78,8 \\
G-38- Teste não Verbal de Inteligência & 41 & 78,8
\end{tabular}

PMK

CAT - A (suplemento)

Bateria Fatorial CEPA

Teste Raven de Operações Lógicas

Teste das Pirâmides das Cores-14M

Matrizes Progressivas Coloridas

WAIS

Escala de Inteligência Stanford-Binet

D-48

$16 \mathrm{PF}-5^{\mathrm{A}}$. edição

DAT

INV

Pré-Bender

Cubos de Kohs

Inventário de Interesses Angelini e Thurstone

Teste das Fábulas

Benton

Escala de Beck

Kuder - Inventário de Interesses

Test Guest Visomotor

Fábulas de Düss

Teste Palográfico

Teste das Pirâmides das Cores-24M

Teste de Prontidão para Leitura

Bender Hutt

UP

Teste das Cores

Escala de Gesell

WPPSI

Teste de Goodenough

CIA

$\mathrm{AC}$

Teste Metropolitano de Prontidão

Inventário de Sintomas de Stress - ISSL

IDATE

Teste de Organização Percepto-Motora

D-70

Teste Piaget-Head

R-1

Teste de Aptidão Mecânica

IDATE-C

Questionário Vocacional de Interesses

IFP - Inventário Fatorial de Personalidade

Inventário Profissional de Interesse

Teste de Reproduções de Figuras

GEIST - Inventário llustrado de Interesses

Escala de Stress Infantil

Questionário Desiderativo

ACRE

Bateria de Testes de Aptidão Geral

IAR

R-2

Teste de Prontidão para Alfabetização

Inventário de Atitudes de Trabalho

BBT

Teste Diagnóstico de Habilidade pré-escolar

Teste de Maturidade para Leitura

Escala de Maturidade para a Escolha Profissional 12

AC 15

Diagnóstico Organizacional

Teste de Desempenho Escolar
78,8

76,9

75

73,1

73,1

73,1

71,1

67,3

67,3

63,5

63,5

61,5

59,6

59,6

57,7

55,8

53,8

51,9

51,9

48,1

46,2

46,2

46,2

44,2

42,3

42,3

40,4

40,4

40,4

38,5

38,5

36,5

36,5

36,5

34,6

34,6

34,6

34,6

32,7

30,8

30,8

30,8

30,8

28,8

28,8

26,9

26, 9

26,9

26,9

25

25

25

23, 1

23,1

23, 1

23,1

23, 1

23,1

21, 2

21, 2

21,2 
Como chefiar?

Questionário de Personalidade de Dadahie

QUATI

BPR-5

Phillipson - Teste de Relações Objetais

Tais dados estão presentes em outros estudos de natureza semelhante, como o proposto por Alves, Alchieri e Marques (2001), em que T.A.T., Raven, WISC, HTP, C.A.T., Bender e Rorschach aparecem como os testes mais ensinados em 64 cursos de graduação. Por outro lado, no trabalho desenvolvido por Vasconcelos e Toledo de Santana (2001), além de Raven, HTP, Desenho da Figura Humana, T.A.T. e Rorschach, dois outros instrumentos que tiveram porcentagens pequenas no presente estudo, apareceram como parte do conteúdo das disciplinas na Universidade Federal da Paraíba (INV e LIP).

Comparando os resultados dos grupos I e II, observa-se que, da relação de 15 instrumentos mais conhecidos, dois do grupo I (C.A.T. animais suplemento e Matrizes Progressivas Coloridas) não aparecem na lista do grupo II, e vice-versa (Rorschach e WISC III). O destaque vai para o Rorschach que é o segundo mais conhecido $(92,3 \%)$ entre os sujeitos formados e que não figura entre os mais conhecidos entre alunos último anistas; tal fato certamente se explica pela ausência desses testes na formação dos sujeitos.

Em relação aos instrumentos menos pontuados, os que seguem não foram identificados por nenhum sujeito do grupo de estudantes: Lendo e Escrevendo, STAXI Inventário de Expressão de Raiva Traço-estado, Teste Prontidão Horizontes, VIG - Bateria Burocrática, Escala de Avaliação do Comportamento Infantil para Professor, Escala Fatorial de Ajustamento EmocionalNeuroticismo e Teste de Quadros para Adolescentes. Já no grupo de psicólogos, três outros instrumentos não foram identificados: OPK - Teste de Agradabilidade Básica, Coordenação Bi-manual e Teste Locus de Controle - Rotter.

Quanto ao segundo objetivo do estudo (identificar os instrumentos mais utilizados pelos sujeitos), os resultados estão apresentados na Tabela 3. Os instrumentos mais utilizados são também os instrumentos mais conhecidos pelos estudantes de uma maneira geral. A seguir encontra-se a seqüência dos instrumentos mais utilizados e entre parênteses apresenta-se a posição em relação aos instrumentos mais conhecidos: Teste de Apercepção Temática - T.A.T. (1), O Desenho da Fi- gura Humana (3), Teste de Zulliger (9), Bender - Teste Gestáltico Viso-Motor (4), Teste da Árvore (11), C.A.T. A (6), Escala de Maturidade Mental Columbia (10), C.A.T. H (8), C.A.T. S (11), Matrizes Progressivas escala geral (6), Matrizes Progressivas - escala avançada (2), Teste de Wartteg (5), Teste das Fábulas (16), M.M.P.I. (11) e Matrizes Progressivas Coloridas (11).

\section{Tabela 3: Freqüência e respectiva porcentagem dos instrumentos mais utilizados por estudantes de Psicologia.}

\begin{tabular}{|c|c|c|}
\hline Nomes dos Instrumentos & $\mathbf{F}$ & $\%$ \\
\hline Teste de Apercepção Temática - T.A.T. A & 50 & 60,98 \\
\hline O Desenho da Figura Humana & 44 & 53,66 \\
\hline Teste Zulliger & 43 & 52,44 \\
\hline Bender - Teste Guestáltico Visomotor & 43 & 52,44 \\
\hline Teste da Árvore & 43 & 52,44 \\
\hline Teste de Apercepção Temática C.A.T. A & 31 & 37,8 \\
\hline Escala de Maturidade Mental Columbia - CEPA & 28 & 34,15 \\
\hline Teste de Apercepção Temática C.AT. H & 27 & 32,93 \\
\hline Teste de Apercepção Temática C.A.T.A - suplem. & 23 & 28,05 \\
\hline Raven - escala geral & 23 & 28,05 \\
\hline Raven - escala avançada & 21 & 25,61 \\
\hline Wartegg & 19 & 23,17 \\
\hline Teste das Fábulas & 19 & 23,17 \\
\hline MMPI & 19 & 23,17 \\
\hline Matrizes Progressivas Coloridas & 16 & 19,51 \\
\hline Escala Maturidade Mental Columbia - Casa Psic. & 16 & 19,51 \\
\hline Fábulas de Düss & 15 & 18,29 \\
\hline WISC & 10 & 12,2 \\
\hline Teste Palográfico & 9 & 10,98 \\
\hline BPR-5 & 9 & 10,98 \\
\hline $16 \mathrm{PF}-5^{\mathrm{a}}$ edição & 9 & 10,98 \\
\hline AC - Atenção Concentrada & 9 & 10,98 \\
\hline Bateria Fatorial CEPA & 8 & 9,76 \\
\hline Pré-Bender & 7 & 8,54 \\
\hline IAR & 7 & 8,54 \\
\hline IAT Inventário Atitudes para o Trabalho & 7 & 8,54 \\
\hline PMK & 7 & 8,54 \\
\hline Teste Raven de Operações Lógicas & 7 & 8,54 \\
\hline LIP - Levantamento de Interesses Profissionais & 6 & 7,32 \\
\hline Bender Hutt & 6 & 7,32 \\
\hline Teste Piaget-Head & 5 & 6,1 \\
\hline G36 Teste não Verbal d Inteligência & 5 & 6,1 \\
\hline Rorschach & 4 & 4,88 \\
\hline Teste de Organização Percepto-Motora & 4 & 4,88 \\
\hline ACRE & 4 & 4,88 \\
\hline G38 Teste não Verbal de Inteligência & 4 & 4,88 \\
\hline WISC III & 4 & 4,88 \\
\hline Inventário de Interesses Angelini e Thurstone & 3 & 3,66 \\
\hline Teste de Aptidão Mecânica & 3 & 3,66 \\
\hline Teste de Goodenough & 3 & 3,66 \\
\hline IDATE & 2 & 2,44 \\
\hline Questionário de Orientação Individual & 2 & 2,44 \\
\hline INV & 2 & 2,44 \\
\hline AC15 - Teste de Atenção Concentrada & 2 & 2,44 \\
\hline Teste Metropolitano de Prontidão & 2 & 2,44 \\
\hline
\end{tabular}


Teste das Pirâmides das Cores - 24M - Pfister 2

Diagnóstico Organizacional

Escala de Gesell

IFP - Inventário Fatorial de Personalidade Inventário de Sintomas de Stress de LIPP Inventário Profissional de Interesses

D70

Escala de Intelig. Stanford-Binet (Terman-Merrill) D48

Kuder - Inventário de Interesses

Teste das Cores

Prontidão de Leitura

RF - Reprodução de Leituras

Benton - Teste de Retenção Visual

BTAG - Bateria de Testes de Aptidão Geral

BTBC - Bateria dos Conceitos Básicos de Bohen

CIA

Cubos de Kohs

EDDP - Escore Deteriorização Desenho da Pessoa1

MM - Teste as Minhas Mãos

Teste de Maturidade para Leitura

APO - Área, Profissões e Objetos

EMEP -Escala de Maturidade Escolha Profissional

Escalas de Personalidade de Comrey

Kit brinquedos e brincadeiras para o bebê

Os contos de fada e a psicopedagogia

QUATI - Questionário de Avaliação Tipológica

QVI - Questionário Vocacional de Interesses

R-1 Teste não Verbal de Inteligência

R-2 Teste não Verbal de Inteligência

RLN Raciocínio Lógico Numérico

TC Teste de Destreza Digital

TEPEM Teste de Prontidão Emocional para Motorista1

Teste dos Relógios

Teste Prontidão para Alfabetização

Teste das Pirâmides das Cores 14M - Pfister

ADT Inventário de Administração de Tempo

Questionário Desiderativo

Coordenação Bi-manual Edites

Relações Lógicas - raciocínio lógico

D2 Teste de Atenção Concentrada

MTB série Both de testes manuais

Escala de Beck

Teste de Symonds

Teste de Szondi

WAIS Escala de Inteligência Wechsler para Adultos 1

WPPSI Escala Wechsler de Inteligência Pré-escolares 1

TAA Teste de Aptidão Acadêmica

Test de Luscher

TIPITI exame de linguagem

Teste de Liderança Situacional

Quanto aos resultados do grupo II, eles possuem as mesmas características observadas no grupo I, ou seja, os instrumentos mais conhecidos também aparecem como os mais utilizados. Os dados estão apresentados na Tabela 4 e a seguir encontram-se os mais citados: WISC (1); O Desenho da Figura Humana (4); Teste de Apercepção Temática (8); Escala de Maturidade Men- tal Columbia - CEPA (15); Teste de Wartegg (9); C.A.T. humanas (11); Teste da Árvore (12); Bender (7); RavenMatrizes Progressivas - escala geral (5); Bateria Fatorial CEPA (21); Raven - Matrizes Progressivas -escala avançada (6); C.A.T. animais (10); M.M.P.I. (14), Rorschach (2) e Inventário de Interesses Angelini e Thurstone. Vale destacar que os valores entre parênteses indicam as posições dos instrumentos mais conhecidos pelos psicólogos.

\section{Tabela 4: Freqüência e respectiva porcentagem dos instrumentos mais utilizados por psicólogos.}

\begin{tabular}{|c|c|c|}
\hline Nomes dos Instrumentos & $\mathbf{F}$ & $\%$ \\
\hline WISC & 28 & 53,8 \\
\hline O Desenho da Figura Humana & 28 & 53,8 \\
\hline Teste de Apercepção Temática & 26 & 50 \\
\hline Escala de maturidade Mental Columbia - CEPA & 26 & 50 \\
\hline Teste de Wartegg & 25 & 48,1 \\
\hline C.A.T. humanas & 23 & 44,2 \\
\hline Teste da Árvore & 23 & 44,2 \\
\hline Bender & 21 & 40,4 \\
\hline Raven - Matrizes Progressivas - escala geral & 21 & 40,4 \\
\hline Bateria Fatorial CEPA & 20 & 38,5 \\
\hline \multicolumn{2}{|l|}{ Raven - Matrizes Progressivas - escala avançada19 } & 36,5 \\
\hline C.A.T. animais & 18 & 34,6 \\
\hline M.M.P.I. & 16 & 30,8 \\
\hline Rorschach & 15 & 28,8 \\
\hline Inventário de Interesses Angelini e Thurstone & 15 & 28,8 \\
\hline Escala de Maturidade Mental Columbia -Casa do Psi & c.14 & 26,9 \\
\hline Pré-Bender & 14 & 26,9 \\
\hline PMK & 13 & 25 \\
\hline Matrizes Progressivas Coloridas & 13 & 25 \\
\hline WISC III & 13 & 25 \\
\hline G-36 & 12 & 23,1 \\
\hline Zulliger & 11 & 21,1 \\
\hline $16 \mathrm{PF}$ & 11 & 21,1 \\
\hline G-38 & 11 & 21,1 \\
\hline Teste Palográfico & 10 & 19,2 \\
\hline WAIS & 10 & 19,2 \\
\hline D-48 & 10 & 19,2 \\
\hline Kuder & 10 & 19,2 \\
\hline INV & 10 & 19,2 \\
\hline DAT & 9 & 17,3 \\
\hline Fábulas de Düss & 9 & 17,3 \\
\hline Test Guest Visomotor & 9 & 17,3 \\
\hline Escala de Inteligência Stanford-Binet & 8 & 15,4 \\
\hline Teste Piaget-Head & 8 & 15,4 \\
\hline UP & 8 & 15,4 \\
\hline Teste Metropolitano de Prontidão & 7 & 13,5 \\
\hline Teste de Goodenough & 7 & 13,5 \\
\hline Teste de Prontidão para Leitura & 7 & 13,5 \\
\hline Questionário Vocacional de Interesse & 7 & 13,5 \\
\hline Teste das Fábulas & 6 & 11,5 \\
\hline C.A.T. Animais suplemento & 6 & 11,5 \\
\hline $\mathrm{ClA}$ & 6 & 11,5 \\
\hline
\end{tabular}


Teste Raven de Operações Lógicas

Cubos de Kohs

Teste das Cores

Teste de Organização Percepto-Motora

Teste de Prontidão para Alfabetização

Teste das Pirâmides das Cores - 14M

Teste das Pirâmides das Cores - 24M

IAT

IFP

D-70

Escalas de Beck

Benton

Teste Diagnóstico de Habilidade Pré-escolar

Teste de Aptidão Mecânica

ACRE

$\mathrm{R}-1$

QUATI

AC 15

Escala de Maturidade para Escolha Profissional

Teste de habilidade para o Trabalho Mental

Questionário Confidencial

Teste Eqüicultural de Inteligência - escala 2

Diagnóstico Organizacional

Escala de Gesell

Inventário de Expectativas sobre o Álcool

BPR-5

Inventário Profissional de Interesses

Teste de Rapidez Numérica

Bender Hutt

WPPSI

Teste de Apercepção para Idosos

IAR

Teste de Compreensão Mecânica

Inventário de Interesses Bessa-Tramer

Lista de problemas Pessoais de Mooney

Como chefiar?

EDDP

GEIST

IDATE

Teste de Reproduções de Figuras

TIPITI

Escala Fatorial de Ajustamento em.-neuroticismo

Teste de Symonds

Teste de Maturidade para Leitura

$\mathrm{PHD}$

Inventário de Sintomas de Stress - ISSL

O desenvolvimento do comportamento no 1 ano

Teste de Rapidez Numérica

Bateria de Testes de Aptidão Geral

Cornell Index

Escala de Preconceito Profissional

IDATE C

Teste de Nível Mental

Questionário de orientação Individual

Teste Eqüicultural de Inteligência - escala 3

Teste de Sondagem Intelectual

Teste Projetivo Ômega

AC

Área, Profissões e Objetos

Coleção Papel de Carta

Escalas de Personalidade de Comrey
Lendo e Escrevendo

Questionário Íntimo

R-2

Raciocínio Lógico-Numérico

SSO de Liderança

Teste de Destreza Digital

Teste Becasse de Maturidade Escolar

Teste de Mandala de Palavras

Teste Projetivo Sonoro

Teste de Estruturas Vocacionais

Inventário de Administração do Tempo

Escala de Stress Infantil

Figuras Complexas de Rey

Questionário de Saúde Geral

Questionário Desiderativo

Teste do Desenho de Silver

Teste de Desempenho Escolar

Bateria TSP

BBT

Escala de Hamilton

Teste de Relações Objetais

Teste de Szondi

Diagnóstico do Desenvolvimento

Diagnóstico Tipológico Organizacional

Teste de Atenção Difusa

Test de Luscher

Teste de Quadros para Adolescentes

1,9

1,9

1,9

1,9

1,9

1,9

1,9

1,9

1,9

1,9

1,9

1,9

1,9

1,9

1,9

1,9

1,9

1,9

1,9

1,9

1,9

1,9

1,9

1,9

1,9

1,9

1,9

Comparando os dois grupos, é possível observar que muitos dos instrumentos aparecem como os mais utilizados em ambos os grupos, exceto os seguintes que aparecem como mais freqüentes em apenas um: Teste de Zulliger, C.A.T. animais suplemento, Teste das Fábulas e Matrizes Progressivas Coloridas (grupo I); WISC, Bateria Fatorial CEPA e Rorschach (grupo II).

\section{Discusș̃̃o}

O presente estudo teve como objetivo identificar os instrumentos mais conhecidos e utilizados pelos psicólogos e por estudantes de psicologia. Constatou-se, a partir da análise dos dados, que os instrumentos mais conhecidos são também os mais utilizados, na grande maioria das vezes, com alguma variação na seqüência apresentada em uma ou outra situação, por ambos os grupos.

Tal constatação, já esperada, confirma a tendência de se reproduzir o conhecido e dominado, e a falta de abertura para o novo. Por mais que isso esteja presente em diferentes áreas de conhecimento, a consequiência é complicada e desastrosa, pois se perpetua o ensino das mesmas técnicas e não se possibilita que concepções mais recentes sejam integradas à prática profissional, sem que, por outro lado, as boas técnicas antigas 
sejam valorizadas. Almeida (1999) aponta que o fraco diálogo entre investigadores e profissionais impede o avanço dos testes psicológicos, e que o desenvolvimento deste instrumental está fortemente associado ao desenvolvimento do país, portanto países em ascensão tendem a oferecer melhores e mais novos materiais.

Outro dado merece atenção. A diferença encontrada entre os grupos no que diz respeito ao conhecimento dos instrumentos, revelou-se pequena, o que remete àquela discussão que evidencia que apenas uma parcela da comunidade de psicólogos se atualiza e continua a estudar, enquanto grande parte se satisfaz com os conhecimentos adquiridos na graduação.

Pesquisas futuras poderiam ser realizados a fim de se levantar a qualidade dos instrumentos que estão sendo ensinados nos vários cursos de graduação nas universidades brasileiras, pois como sugere Castro (2001) a avaliação sobre os elementos inerentes ao processo ensinoaprendizagem de disciplinas de avaliação psicológica, é sempre oportuna, uma vez que pode gerar reflexões sobre estratégias de ensino, de forma a valorizar e consolidar o papel das técnicas na formação do psicólogo. No estudo desenvolvido por Wechsler e cols. (2000) entre estudantes universitários, com o objetivo de identificar as necessidades de pesquisa em avaliação psicológica, WISC, Bender, Columbia, HTP e Rorschach foram considerados os testes que mais necessitam de pesquisas no Brasil. Certamente, os sujeitos se basearam nos instrumentos ensinados nas suas respectivas formações profissionais para poder avaliar, o que pode sugerir que o universo de conhecimento é também restrito e parcial.

\section{REFERÊNCIAS}

Alchieri, J. C., \& Scheffel, M. (2000). Indicadores da produção científica brasileira em avaliação psicológica: resultados da elaboração de uma base de dados dos artigos publicados em periódicos brasileiros de 1930 a 1999. Anais do $\checkmark$ Encontro Mineiro de Avaliação Psicológica teorização e prática e VIII Conferência Internacional Avaliação Psicológica - formas e contextos. PUC Minas, Belo Horizonte/MG, 99-100.

Almeida, L. S. (1999). Avaliação psicológica - exigências e desenvolvimentos nos seus métodos. Em S. M. Wechsler \& R. S. L. Guzzo (Orgs.) Avaliação Psicológica-perspectiva internacional (pp. 41-55). São Paulo: Casa do Psicólogo.
Em contrapartida, não é exeqüível que muitos ou todos os instrumentos sejam discutidos ao longo da graduação, tendo em vista que o número de instrumentos não permite e que a prioridade é a qualidade de ensino, em detrimento da quantidade de técnicas. Em relação ao tema, Alves (2001) procurou avaliar, de maneira ampla, o ensino das técnicas de exame psicológico. O estudo revelou que os professores ensinam mais instrumentos do que é considerado como básico ou mínimo pelos próprios e que há pouca diferença entre a lista de instrumentos psicológicos efetivamente ensinados e a lista daqueles que foram considerados como mais indicados para o ensino e, sob esta perspectiva, erroneamente poderia se dizer que o ensino em avaliação psicológica está satisfatório.

Para Pasquali (1999) o descrédito que ainda se encontra atualmente em relação aos instrumentos psicológicos, muito se deve à deficiente formação na área. $\mathrm{O}$ autor acredita que, embora a pesquisa na área ainda seja incipiente, os poucos pesquisadores que estão trabalhando, de alguma forma, já estão incomodando a classe de psicólogos, no que se refere ao problema da instrumentalização, da qualidade, do uso e da melhoria de testes psicológicos.

O presente estudo constituiu-se num trabalho de levantamento e, embora este tipo de metodologia ainda receba críticas, mudanças se fazem a partir da reflexão de conjuntos de dados que revelam os diferentes estados das áreas de conhecimento. Portanto, estudos desta natureza são ainda importantes na área de avaliação psicológica para que as mudanças aconteçam e sejam pautadas nos dados já estudados.

Almeida, L. S., Prieto, G., Muñiz, J., \& Bartram, D. (1998). O uso dos testes em Portugal, Espanha e países Iberoamericanos. Psychologica, 20, 27-40.

Alves, I. C. B., Alchieri, J. C., \& Marques, K. (2001). Panorama Geral do Ensino das Técnicas de Exame Psicológico no Brasil. I Congresso de Psicologia Clínica Programas e Resumos. Universidade Presbiteriana Mackenzie, São Paulo, 10-11.

Alves, I. C. B. (2001). O ensino das Técnicas de Exame Psicológico de Acordo com os Professores. Anais do IV Encontro da Sociedade Brasileira de Rorschach e outras técnicas de avaliação psicológica. Universidade São Francisco, Itatiba-SP, 62.

Azevedo, M. M., Almeida, L. S., Pasquali, L., \& Veiga, H. M. S. (1996). Utilização dos testes psicológicos no Brasil: da- 
dos de estudo preliminar em Brasília. Em L. S. Almeida e cols. Avaliação Psicológica: formas e contextos v. IV (pp. 213-219). Braga, Portugal.

Buettner, G. E. V. (1997). Por que a formação continuada? In CRP - Jornal do CRP, ano 17 (107), 2-4.

Cardoso, S. M. V. (1994). A prática docente no ensino superior particular noturno: um estudo de caso. Tese de Doutorado em Educação, Universidade Estadual de Campinas, Campinas.

Castro, P. F. (2001). Reflexões acerca do processo ensino-aprendizagem no método Rorschach na realidade brasileira. Anais do IV Encontro da Sociedade Brasileira de Rorschach e outras técnicas de avaliação psicológica. Universidade São Francisco, Itatiba-SP, 59-60.

Hays, R., \& Wellard, R. (1998). In training assessment in postgraduate training for general practice. Medical Education, 32 (5), 507-513.

Jacquemin, A. (1995). Ensino e pesquisa sobre testes psicológicos. Boletim de Psicologia, XLV (102), 19-21.

Kroeff, P. (1998). Síntese de posicionamentos a serem feitos quanto ao uso de testes psicológicos em Avaliação Psicológica. Anais da $18^{a}$ Reunião Anual de Psicologia. Sociedade de Psicologia de Ribeirão Preto, 535-537.

Noronha, A. P. P. (1999). Avaliação Psicológica: usos e problemas com ênfase nos testes. Tese de Doutorado, Instituto de Psicologia e Fonoaudiologia, PUC-Campinas.

Pasquali, L. (1999). Instrumentos Psicológicos: manual prático de elaboração. LabPAM/ IBAPP, Brasília.

Pfromm Netto, S. (1991). Psicologia e guia de estudo. E.P.U: São Paulo.

Primi, R., \& Munhóz, A. M. H. (1998). Um estudo sobre proficiência na disciplina Técnicas de Exame Psicológico (TEP). Psico-Usf, 3 (2), 75-86.
Rocha Jr., L., \& Sá, A. A. (1997). Currículos de Psicologia: uma análise crítica. Anais do I Encontro sobre Psicologia Clínica. Universidade de Mackenzie, 145-147.

Sisto, F. F., Sbardelini, E. T. B., \& Primi, R. (2001). Contextos e questões da avaliação psicológica. São Paulo: Casa do Psicólogo.

Vasconcelos, Z. B., \& Toledo de Santana, C. M. (2001). O Ensino das Técnicas de Exame Psicológico na Universidade Federal da Paraíba. I Congresso de Psicologia Clínica-programas e resumos. Universidade Presbiteriana Mackenzie, São Paulo, 207-208.

Wechsler, S. M., Siqueira, L. G. G., Reis, C. L., Barbosa, N. C., Schelini, P. W., Nakano, T. C., Kodama, M. C., Merlin, M. S., Costa, A. C. G., Leal, M. G., Nogueira, C. M., Zia, K. P., Rodrigues, R. R., \& Reani, P. R. T. (2000). Percepção da Necessidade de Pesquisas em Avaliação Psicológica. Anais do V Encontro Mineiro de Avaliação Psicológica e VIII Conferência Internacional de Avaliação Psicológica. PUC Minas, Belo Horizonte, 53.

Witter, G.P., Witter, C., Yukmitsu, M. T. C. P., \& Gonçalves, C. L. C. (1992). Atuação do psicólogo escolar e educacional no Brasil: perspectivas através de textos (1980-1992) Em A. L. Francisco., C. R. Klomfahs., N. M. D. Rocha (Orgs.). Conselho Federal de Psicologia - Psicólogo Brasileiro construção de novos espaços (pp.23-53). São Paulo: Átomo.

Witter, C. (1996). Psicologia escolar: produção cient[ifica, forma;áo e atuação (1990-1994). Tese de Doutorado. Universidade de São Paulo. São Paulo.

Recebido em: 02/05/02

Revisado em: 16/06/02

Aprovado em: 29/10/02 